\title{
The variability of the spectrum of Arakelian 120. II - Evidence for a small broad line emitting region
}

\section{Citation}

Peterson, B. M., K. A. Meyers, E. R. Carpriotti, C. B. Foltz, B. J. Wilkes, and H. R. Miller. 1985. "The Variability of the Spectrum of Arakelian 120. II - Evidence for a Small Broad Line Emitting Region." The Astrophysical Journal 292 (May): 164. doi:10.1086/163142.

\section{Published Version}

$10.1086 / 163142$

\section{Permanent link}

http://nrs.harvard.edu/urn-3:HUL.InstRepos:32955252

\section{Terms of Use}

This article was downloaded from Harvard University's DASH repository, and is made available under the terms and conditions applicable to Other Posted Material, as set forth at http:// nrs.harvard.edu/urn-3:HUL.InstRepos:dash.current.terms-of-use\#LAA

\section{Share Your Story}

The Harvard community has made this article openly available.

Please share how this access benefits you. Submit a story.

\section{Accessibility}


The Astrophysical Journal, 292:164-171, 1985 May 1

(c) 1985. The American Astronomical Society. All rights reserved. Printed in U.S.A.

THE VARIABILITY OF THE SPECTRUM OF ARAKELIAN 120. II. EVIDENCE FOR A SMALL BROAD LINE EMITTING REGION

\author{
B. M. Peterson, K. A. Meyers, and E. R. Capriotti \\ C. B. FolTz \\ Department of Astronomy, University of Illinois \\ B. J. WILKES \\ Center for Astrophysics, Smithsonian Astrophysical Observatory \\ AND \\ H. R. MILLER ${ }^{1}$ \\ Department of Physics and Astronomy, Georgia State University \\ Received 1984 August 30; accepted 1984 November 12
}

Perkins Observatory, The Ohio State and Ohio Wesleyan Universities; and Department of Astronomy, Ohio State University

\begin{abstract}
Four years of spectroscopic and photometric monitoring of the Seyfert 1 galaxy Akn 120 reveal that the broad $\mathrm{H} \beta$ line flux changes on a time scale indistinguishable from that of the optical continuum, which sets an upper limit of $\sim 30$ lt-day on the size of the broad-line region. The high luminosity of the Akn 120 continuum source thus implies a very high radiation parameter, significantly larger than the values used in successful models of the emission-line region, even if the electron density is of order $10^{9} \mathrm{~cm}^{-3}$, which is the maximum allowable from C III] $\lambda 1909$ line intensity considerations. It is suggested that the radiation parameter is in fact consistent with those used in models, because the electron density is rather higher than the currently preferred value of $10^{9} \mathrm{~cm}^{-3}$. Consequently, the $\mathrm{C} \mathrm{III]} \lambda 1909$ emission must arise in a different region than the Balmer lines, which would be consistent with the IUE results for NGC 4151. It is also suggested that the slight redward displacement of $\mathrm{H} \beta$ relative to the systemic redshift may be due to a gravitational redshift, which implies the existence of a massive object in the nucleus of Akn 120.
\end{abstract}

Subject headings: galaxies: individual - galaxies: Seyfert - relativity

\section{INTRODUCTION}

Arakelian 120 is a luminous Seyfert 1 galaxy which exhibits large-amplitude variability in the optical continuum and in the permitted-line spectrum. On the basis of archive plates of the Harvard College Observatory, Miller (1979) has shown that its optical-continuum variability extends back to at least 1929 and that the amplitude of the variations is greater than 2 mag in $B$. The variability of the optical permitted-line spectrum was discovered independently by three groups (Foltz et al. 1981; Kollatschny et al. 1981a; Schulz and Rafanelli 1981), and the spectrum has been monitored fairly closely since this initial discovery (Peterson et al. 1983; hereafter Paper I).

It has been suggested that the variability of the permittedline fluxes and profiles is attributable to light-travel-time effects (Capriotti, Foltz, and Peterson 1982); i.e., because of the finite spatial extent of the broad-line-emitting region (BLR), there is a time delay between a change in the ionizing continuum and a corresponding change in the emission-line flux. An external observer does not, of course, observe continuum pulses as spherical shells propagating through the BLR, but rather observes the pulses as paraboloids, which are loci of constant time delay. Given sufficient data on the continuum light curve and the time dependence of the permitted-line profiles, it is in principle possible to determine the kinematic structure of the BLR (Blandford and McKee 1982). It is the goal of this monitoring project to study the gross characteristics of the

\footnotetext{
${ }^{1}$ Visiting Astronomer, Kitt Peak National Observatory and Lowell Observatory.
}

BLR kinematics. By examining the changes in the permittedline profiles as the continuum varies, it should be possible to determine whether radial outflow, infall, turbulent, or rotational models of the BLR are appropriate.

In Paper I, it was pointed out that Akn 120 is an excellent candidate for a study of this nature because of its apparent and absolute brightness, strong broad lines, and large-amplitude variability. The results of Paper I showed that the broad $\mathrm{H} \beta$ line appears to respond very quickly to optical-continuum changes. This pointed out the need for closely spaced spectral observations of this source.

In an attempt to establish the time scale for response of the BLR to changes in the optical continuum, spectra of Akn 120 were obtained at least once per month between 1983 September and 1984 April. In this contribution, the rapid response of the broad $\mathrm{H} \beta$ feature to continuum changes is established. It is concluded that most of the BLR gas lies within 30 lt-day $(0.026 \mathrm{pc})$ of the continuum source. This presents some major difficulties in terms of our understanding of the physics of the BLR, and some of these problems are discussed in the last section of this paper.

\section{OBSERVATIONS}

\section{a) Spectroscopic Observations}

Most of the spectra discussed here were obtained with the Ohio State University image dissector scanner (IDS; Byard et al. 1981) on the Perkins $1.8 \mathrm{~m}$ reflector of Ohio Wesleyan University and Ohio University at Lowell Observatory. Additional spectra were obtained with the photon-counting Reticon 
systems on the Steward Observatory $2.3 \mathrm{~m}$ telescope on Kitt Peak and the $\mathrm{MMT}^{2}$ on Mount Hopkins. All three detectors are dual-channel devices, which allows nearly contemporaneous measurement of the night-sky spectrum. The wavelength-dependent sensitivity of the detectors is calibrated by observations of standard stars. Absolute spectrophotometry was not attempted, however, since many of these observations were made under conditions of variable transparency. Details of the data reduction procedures will be discussed in a future paper.

A journal of observations appears in Table 1 . The UT date and Julian date of the observations are given in columns (1) and (2). The telescope and detector are listed in columns (3) and (4). The projected aperture geometry is shown in column (5). Differences in aperture geometry should have a negligible effect on these observations since the line-emitting regions and active nucleus are unresolved on scales of order 1" (Whittle 1981) and the underlying galaxy is faint relative to the nucleus; under these conditions aperture effects will be very small (Peterson and Collins 1983).

The spectra from early 1983 were obtained too late for inclusion in Paper I, and have since been discussed elsewhere (Foltz, Wilkes, and Peterson 1983; Meyers and Peterson 1985).

An enlargement of the region around $\mathrm{H} \beta$ is shown in Figure 1 for one of our spectra (note that the zero point and flux scale are arbitrary). The straight lines in this figure show the continuum placement for the line measurements.

The flux in the [O III] $\lambda \lambda 4959,5007$ lines is measured by drawing a straight line underneath these features, which are superposed on a broad shelf of emission redward of $\mathrm{H} \beta$. The flux of $\mathrm{H} \beta$ and its rest-frame $(z=0.0325)$ equivalent width are measured by connecting the two minima between the $\mathrm{Fe}$ II $\lambda 4570$ blend and $\mathrm{H} \beta$ and between $\mathrm{H} \beta$ and the $\mathrm{Fe}$ II $\lambda 5190$ blend

${ }^{2}$ The Multiple Mirror Telescope (MMT) is a joint facility of the University of Arizona and the Smithsonian Institution. with a straight line which approximates a nearly flat power law. The $[\mathrm{O}$ III] lines and the weak line at $\sim 5050 \AA$ (rest) are excluded from the $\mathrm{H} \beta$ line measurement, but the redward extension of $\mathrm{H} \beta$ is included; even though this shelf is probably attributable to $\mathrm{Fe}$ II $\lambda \lambda 4924,5018$ or [O III] $\lambda \lambda 4959,5007$, or both, from the broad-line region (Foltz, Wilkes, and Peterson 1983; Meyers and Peterson 1985), Meyers and Peterson find that the flux in the shelf is well correlated with the $\mathrm{H} \beta$ flux, so for our purposes inclusion of the shelf makes very little difference as long as the measurements are consistent. The shelf is thus included in the $\mathrm{H} \beta$ measurements to facilitate comparison with earlier work.

The rest-frame equivalent width of $\mathrm{H} \beta, W_{0}(\mathrm{H} \beta)$, is given in column (6) of Table 1 , and the $[\mathrm{O} \mathrm{III}] 25007 / \mathrm{H} \beta$ flux ratio is given in column (7). In contrast to Paper I, the [O III] $\lambda 44959 / \mathrm{H} \beta$ flux ratio is not given. This is because the $\mathrm{H} \beta+$ shelf profile underneath [O III] $\lambda 4959$ cannot be approximated by a straight line, as it can underneath [O III] 25007 . At $4959 \AA$, the intrinsic $\mathrm{H} \beta$ profile is dropping steeply to the red, and the shelf profile is dropping to the blue. The sum of these components leaves a local minimum near $4959 \AA$, which is then partly filled in by narrow [O III] 24959 . If this is not taken into account, the measured $[\mathrm{O} \mathrm{III}] \lambda 5007 /[\mathrm{O} \mathrm{III}] \lambda 4959$ flux ratio is generally larger than predicted by atomic physics. It was therefore decided that only the [O III] $\lambda 5007$ flux would be used. It should be mentioned that the resulting inclusion of a small fraction of the [O III] $\lambda 4959$ flux with the $\mathrm{H} \beta+$ shelf measurements introduces a negligible error in the $\mathrm{H} \beta$ flux because the [O III $] 24959 / \mathrm{H} \beta$ flux ratio is so small (typically $\sim 0.02$ ).

It should also be pointed out that the values for $W_{0}(\mathrm{H} \beta)$ and the $[\mathrm{O}$ III $] \lambda 5007 / \mathrm{H} \beta$ flux ratio given by Foltz, Wilkes, and Peterson (1983) for the 1983 January 9 Reticon spectrum are erroneous - the values given in that paper are actually the measurements for the 1982 December 27 spectrum reported in Paper I. The correct measurements for the 1983 January 9 spectrum are given in Table 1.

TABLE 1

SPECTROSCOPIC OBSERVATIONS

\begin{tabular}{|c|c|c|c|c|c|c|}
\hline $\begin{array}{l}\text { UT Date } \\
\text { (1) }\end{array}$ & $\begin{array}{c}\text { JD } \\
(2,440,000+) \\
(2)\end{array}$ & $\begin{array}{c}\text { Telescope }{ }^{a} \\
\text { (3) }\end{array}$ & $\begin{array}{l}\text { Instrument } \\
\text { (4) }\end{array}$ & $\begin{array}{l}\text { Aperture }^{b} \\
(5)\end{array}$ & $\begin{array}{c}W_{0}(\mathrm{H} \beta) \\
(6)\end{array}$ & 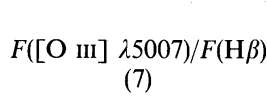 \\
\hline 1983 Jan 8 & 5342 & $1.8 \mathrm{~m}$ & IDS & $5^{\prime \prime}$ & $119.6 \pm 5.7$ & $0.075 \pm 0.007$ \\
\hline 1983 Jan $9 . \ldots \ldots$ & 5343 & $2.3 \mathrm{~m}$ & Reticon & $2.5^{\prime \prime}$ & $87.9 \pm 2.3$ & $0.072 \pm 0.003$ \\
\hline $1983 \operatorname{Jan} 11 \ldots \ldots$ & 5345 & $1.8 \mathrm{~m}$ & IDS & $5^{\prime \prime}$ & $115.7 \pm 5.2$ & $0.082 \pm 0.008$ \\
\hline $1983 \operatorname{Jan} 15 \ldots \ldots$ & 5349 & $1.8 \mathrm{~m}$ & IDS & $5^{\prime \prime}$ & $104.2 \pm 4.6$ & $0.086 \pm 0.007$ \\
\hline 1983 Sep $13 \ldots \ldots$ & 5590 & $1.8 \mathrm{~m}$ & IDS & $7^{\prime \prime}$ & $120.1 \pm 3.4$ & $0.071 \pm 0.004$ \\
\hline 1983 Oct $9 \ldots \ldots \ldots$ & 5616 & $1.8 \mathrm{~m}$ & IDS & $7^{\prime \prime}$ & $112.0 \pm 5.1$ & $0.074 \pm 0.002$ \\
\hline 1983 Oct $10 \ldots .$. & 5617 & $1.8 \mathrm{~m}$ & IDS & $7^{\prime \prime}$ & $103.9 \pm 2.4$ & $0.073 \pm 0.006$ \\
\hline 1983 Nov 7 ....... & 5645 & $1.8 \mathrm{~m}$ & IDS & $7^{\prime \prime}$ & $110.0 \pm 5.2$ & $0.104 \pm 0.008$ \\
\hline 1983 Nov $9 . \ldots \ldots$ & 5647 & $1.8 \mathrm{~m}$ & IDS & $7^{\prime \prime}$ & $103.8 \pm 3.7$ & $0.106 \pm 0.006$ \\
\hline 1983 Nov $29 . \ldots$. & 5667 & $2.3 \mathrm{~m}$ & Reticon & $2.5^{\prime \prime}$ & 102.5 & 0.099 \\
\hline 1983 Dec 5 ...... & 5673 & $1.8 \mathrm{~m}$ & IDS & $7^{\prime \prime}$ & $104.0 \pm 4.2$ & $0.087 \pm 0.006$ \\
\hline $1984 \operatorname{Jan} 4 \ldots \ldots \ldots$ & 5703 & $1.8 \mathrm{~m}$ & IDS & $7^{\prime \prime}$ & $94.5 \pm 3.7$ & $0.079 \pm 0.003$ \\
\hline 1984 Jan 7 ....... & 5706 & $1.8 \mathrm{~m}$ & IDS & $7^{\prime \prime}$ & $85.4 \pm 3.8$ & $0.085 \pm 0.004$ \\
\hline 1984 Jan $28 \ldots \ldots$ & 5727 & $2.3 \mathrm{~m}$ & Reticon & $2.5^{\prime \prime}$ & 79.4 & 0.087 \\
\hline 1984 Feb $4 \ldots \ldots$ & 5734 & $1.8 \mathrm{~m}$ & IDS & $7^{\prime \prime}$ & $90.4 \pm 1.9$ & $0.078 \pm 0.005$ \\
\hline 1984 Feb 6 ...... & 5736 & $1.8 \mathrm{~m}$ & IDS & $7^{\prime \prime}$ & $85.8 \pm 5.3$ & $0.083 \pm 0.012$ \\
\hline 1984 Feb 29 ..... & 5759 & MMT & Reticon & $1^{\prime \prime} \times 2.6^{\prime \prime}$ & $98.0^{-}$ & 0.067 \\
\hline 1984 Mar $3 \ldots \ldots$ & 5762 & $1.8 \mathrm{~m}$ & IDS & $7^{\prime \prime}$ & $103.1 \pm 2.1$ & $0.077 \pm 0.013$ \\
\hline $1984 \operatorname{Mar} 5 \ldots \ldots$ & 5764 & $1.8 \mathrm{~m}$ & IDS & $7^{\prime \prime}$ & $109.3 \pm 3.2$ & $0.079 \pm 0.013$ \\
\hline 1984 Apr 6 ....... & 5796 & $2.3 \mathrm{~m}$ & Reticon & $2.5^{\prime \prime}$ & 122.7 & 0.103 \\
\hline
\end{tabular}

a $1.8 \mathrm{~m}$ is Perkins, $2.3 \mathrm{~m}$ is Steward.

b All apertures round except 1984 Feb 29. 


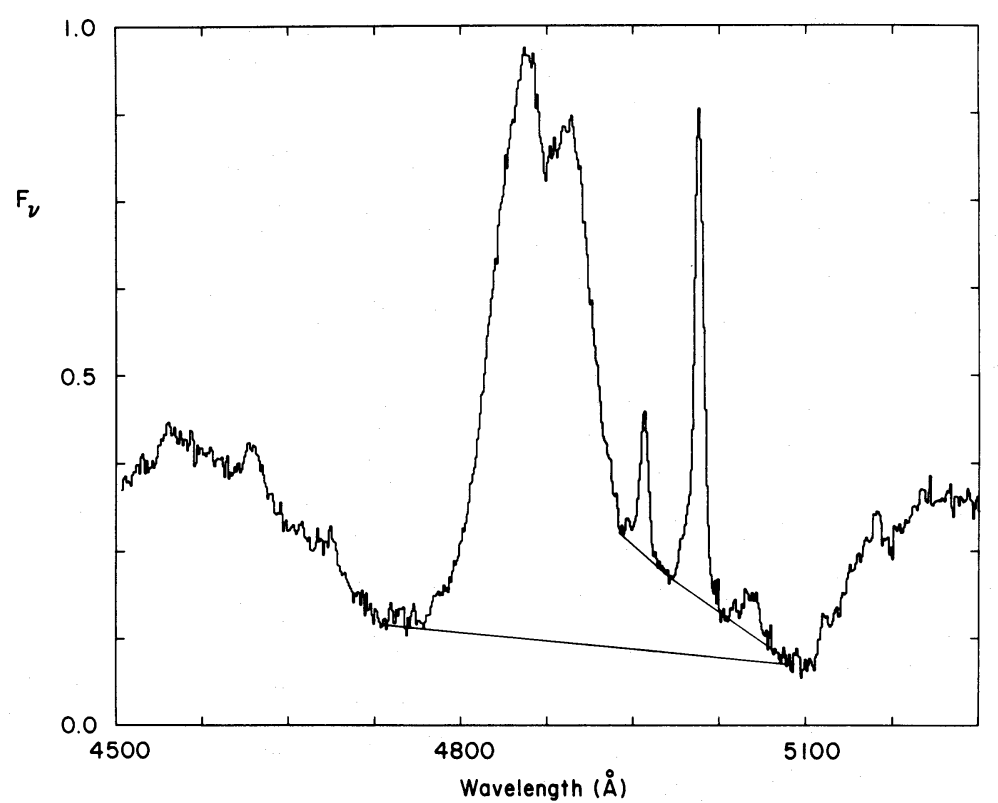

Fig. 1.-An enlargement of the $\mathrm{H} \beta$ region of one of our spectra to demonstrate how the line measurements are made. Note that the zero point and scale on the vertical axis are arbitrary.

\section{b) Photometric Observations}

The spectroscopic observations are complemented by several $U B V$ measurements made with the $0.91 \mathrm{~m}$ telescope at Kitt Peak National Observatory ${ }^{3}$ and the $1.07 \mathrm{~m}$ telescope at Lowell Observatory.

The Automated Filter Photometer, equipped with a 1P21 photomultiplier tube and standard Johnson $U B V$ filters, was employed for the observations obtained at KPNO. The Bowell two-channel photometer, equipped with EMI 6256 and EMI 9526 photomultipliers and standard Johnson $U B V$ filters, was used for the observations obtained at Lowell Observatory. Miller's photoelectric sequence in the field of Akn 120 (Miller 1981) was used for calibration.

The new $U B V$ measurements are presented in Table 2. As in Table 1, the UT date, Julian Date, and telescope appear in columns (1)-(3). The projected diameter of the entrance aperture is given in column (4). The measured values of $V, B-V$, and $U-B$ are given in columns (5), (6), and (7). The formal errors in each bandpass are expected to be at the $1 \%$ level,

\footnotetext{
${ }^{3}$ Kitt Peak National Observatory is operated by the Association of Universities for Research in Astronomy under contract with the National Science Foundation.
}

except for the $V$ measurement on 1983 March 14 which was obtained differentially under non-photometric conditions, and for the $U$ measurements from 1984 March, which are uncertain at about the $4 \%$ level.

As in Paper I, a small correction (about $0.16 \mathrm{mag}$ in $V$ ) has been made to the large-aperture measurements to account for the contribution of the underlying disk of Akn 120. Measurements to which the correction of Paper I has been applied are noted in column (8) of Table 2.

\section{DATA ANALYSIS}

The narrow [O III] $\lambda \lambda 4959,5007$ lines are thought to arise in an extended region. From the narrow-line luminosities and density determinations in Seyfert galaxies, it is easy to show that the extent of the region must be of order $10 \epsilon_{N}{ }^{-1 / 3} \mathrm{pc}$, where $\epsilon_{N}$ is the filling factor for the narrow-line region gas. The fact that the narrow-line regions are partly resolved in some cases (e.g., Walker 1968; Phillips et al. 1983) suggests that dimensions of $100 \mathrm{pc}$ or greater are appropriate. In any case, it is clear that the narrow-line fluxes can be regarded as constant over the time scales of interest here, since the light-travel time across the region is far longer than the time scale for contin-

TABLE 2

$U B V$ MEASUREMENTS

\begin{tabular}{|c|c|c|c|c|c|c|c|}
\hline $\begin{array}{l}\text { UT Date } \\
\text { (1) }\end{array}$ & $\begin{array}{c}\text { JD } \\
(2,440,000+) \\
(2)\end{array}$ & $\begin{array}{c}\text { Telescope }{ }^{a} \\
\text { (3) }\end{array}$ & $\begin{array}{l}\text { Aperture } \\
\text { (4) }\end{array}$ & $\begin{array}{l}V \\
(5)\end{array}$ & $\begin{array}{c}B-V \\
(6)\end{array}$ & $\begin{array}{c}U-B \\
(7)\end{array}$ & $\begin{array}{c}\text { Aperture } \\
\text { Correction Made } \\
\text { (8) }\end{array}$ \\
\hline 1983 Jan 19 & 5353 & $0.91 \mathrm{~m}$ & 15.5 & 13.64 & 0.43 & -0.66 & no \\
\hline 1983 Jan $23 \ldots$ & 5357 & $0.91 \mathrm{~m}$ & 15.5 & 13.54 & 0.46 & -0.74 & no \\
\hline 1983 Mar $14 \ldots \ldots$ & 5407 & $1.07 \mathrm{~m}$ & 24.0 & $13.89+0.15$ & & & yes \\
\hline 1984 Jan $19 \ldots \ldots$ & 5718 & $0.91 \mathrm{~m}$ & 15.5 & 14.08 & 0.40 & -0.71 & no \\
\hline 1984 Mar 1 . ...... & 5760 & $1.07 \mathrm{~m}$ & 24.0 & 13.51 & 0.35 & -0.78 : & yes \\
\hline 1984 Mar 3 ........ & 5762 & $1.07 \mathrm{~m}$ & 24.0 & 13.56 & 0.40 & -0.69 : & yes \\
\hline 1984 Apr $3 \ldots \ldots$ & 5793 & $0.91 \mathrm{~m}$ & 15.5 & 13.79 & 0.45 & -0.58 & no \\
\hline 1984 Apr 4 & 5794 & $0.91 \mathrm{~m}$ & 15.5 & 13.84 & 0.46 & -0.61 & no \\
\hline
\end{tabular}

${ }^{\mathrm{a}} 0.91 \mathrm{~m}$ is KPNO, $1.07 \mathrm{~m}$ is Lowell. 
uum variability. The flux of the narrow [O III $\lambda 25007$ line is thus adopted as a benchmark for calibrating the continuum and broad-line fluxes. This is a commonly adopted procedure (Phillips 1978; Osterbrock and Shuder 1982; Peterson et al. 1982, 1983, 1984).

If the [O III] $\lambda 5007$ flux is constant, then changes in the equivalent width of $[\mathrm{O} \mathrm{III}] \lambda 5007$ are inversely proportional to changes in the optical continuum, and changes in the [O III] $\lambda 5007 / \mathrm{H} \beta$ flux ratio are inversely proportional to changes in the $\mathrm{H} \beta$ flux. Since [O III] $\lambda 5007$ is superposed on the $\mathrm{H} \beta+$ shelf complex, a calculated equivalent width

$$
W_{c}([\mathrm{O} \mathrm{III}])=W_{0}(\mathrm{H} \beta) \frac{F([\mathrm{O} \mathrm{III}] \lambda 5007)}{F(\mathrm{H} \beta)},
$$

where $F([\mathrm{O} \mathrm{III}] \lambda 5007 / F(\mathrm{H} \beta)$ is the $[\mathrm{O} \mathrm{III}] \lambda 5007 / \mathrm{H} \beta$ flux ratio, is used to refer the [O III] equivalent width to the continuum under $\mathrm{H} \beta$. This differs somewhat from $W_{c}([\mathrm{O} \mathrm{III}])$ in Paper $\mathrm{I}$, in which the total [O III] flux was used [i.e., $F\left(\left[\begin{array}{ll}\mathrm{O} & \text { III }]\end{array}\right.\right.$ $\lambda 4959)+F([\mathrm{O}$ III $]$ 25007)]; values from Paper I which are used here are corrected for this difference.

Because the relative flux calibration of the spectra depends so critically on correct measurement of $F([\mathrm{O}$ III $] 25007)$, great care was taken in evaluating the uncertainties. Uncertainties were determined by repeated measurement of $F([\mathrm{O} \mathrm{III}] \lambda 5007) /$ $F(\mathrm{H} \beta)$ and $W_{0}(\mathrm{H} \beta)$ on different occasions, often by different observers. Care was taken to include the highest and lowest reasonable continuum settings. The estimated uncertainties in $F([\mathrm{O} \mathrm{III}] \lambda 5007 / F(\mathrm{H} \beta)$ are almost always less than $\sim 10 \%$ and in $W_{0}(\mathrm{H} \beta)$, for which the continuum placement is rarely ambiguous, are typically $\sim 5 \%$. All detectors used here are dualbeam instruments. By switching the galaxy from one aperture to the other at short intervals, two independent spectra are obtained, and these are reduced separately. Comparison of the two simultaneous spectra thus provides an additional consistency check. The spectra from the independent apertures yield measurements consistent with the errors quoted above. These errors, which are given for most of the individual mea- surements in Table 1 , are consistent with the estimates of Paper I.

In the top half of Figure 2, the continuum magnitudes determined from equation (1) and the measurements listed in Table 1 are plotted as a function of Julian Date. The magnitude scale is set by adopting the measurement of Osterbrock and Phillips (1977) as a zero point, as in Paper I. Note that rms uncertainties are also shown where available. The corresponding $\mathrm{H} \beta$ flux, plotted in magnitudes defined by

$$
m_{\mathrm{H} \beta}=2.5 \log \frac{F([\mathrm{O} \mathrm{III}] \lambda 5007)}{F(\mathrm{H} \beta)},
$$

is shown in the lower half of Figure 2. The overall general agreement of closely spaced data points gives us additional confidence in the integrity of our analysis.

There is, however, one data point in poor agreement with the others. The continuum point of 1983 January 9, obtained with the Steward Reticon, is approximately 0.35 mag higher than the continuum points of 1983 January 8,11 , and 15 obtained with the IDS. This suggests the existence of instrumental differences between the Reticon and the IDS. Aperture effects can be ruled out since the IDS observations employed a larger entrance aperture, which should enhance rather than suppress the continuum relative to the Reticon. To check for instrumental effects, closely spaced observations of Mrk 79 and NGC 5548, which are also being monitored as part of our variability program, were compared. The appropriate measurements are presented in Table 3 , where it is seen that the Reticon and IDS data compare very well. It is also noted that the Reticon observation of 1982 December 27 (Paper I) agrees quite well with the IDS data of 1983 January. It is thus concluded that if there is a systematic difference between the Reticon and the IDS in 1983 January, it is of a nonrecurring nature. It should be emphasized that all 1983 January spectra were reduced from the raw data two or three times and the measurements were made in the same way by all observers. For all observations, the data from the two apertures agree to

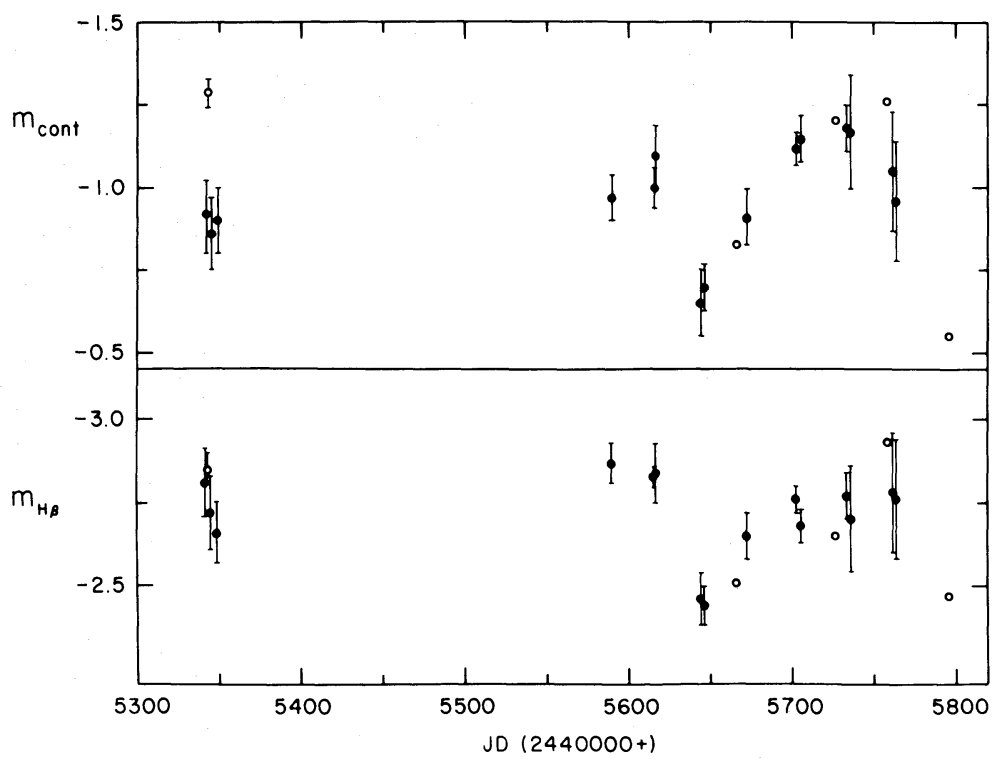

Fig. 2.-Optical continuum and $\mathrm{H} \beta$ magnitudes (on an arbitrary scale) for the data in Table 1 plotted as a function of Julian Date. The closed circles represent data obtained with the IDS, and the open circles represent data from the Reticon scanners. Error bars $(1 \sigma)$ are shown in cases where a large number of independent measurements were made. 
TABLE 3

COMPARISON OF RETICON AND IDS RESUlts FOR Mrk 79 AND NGC 5548

\begin{tabular}{|c|c|c|c|c|c|c|}
\hline $\begin{array}{l}\text { Galaxy } \\
\text { (1) }\end{array}$ & $\begin{array}{l}\text { UT Date } \\
(2)\end{array}$ & $\begin{array}{l}\text { Instrument } \\
\text { (3) }\end{array}$ & $\begin{array}{l}\text { Aperture } \\
\text { (4) }\end{array}$ & $\begin{array}{c}W_{0}(\mathrm{H} \beta) \\
(\AA) \\
(5)\end{array}$ & $\underset{(6)}{F([\mathrm{O} \text { III }] \lambda 4959) / F(\mathrm{H} \beta)}$ & $F\left(\left[\begin{array}{ll}\mathrm{O} & \mathrm{III}] \\
(7) & \lambda 5007) / F(\mathbf{H} \beta)\end{array}\right.\right.$ \\
\hline Mrk 79 & 1984 Jan 30 & Reticon & $2.5^{\prime \prime}$ & 92.2 & 0.119 & 0.401 \\
\hline Mrk 79 & 1984 Feb 4 & IDS & $7^{\prime \prime}$ & 92.2 & 0.119 & 0.428 \\
\hline Mrk 79 & 1984 Feb 6 & IDS & $7^{\prime \prime}$ & 93.4 & 0.121 & 0.415 \\
\hline NGC $5548 \ldots \ldots$. & 1984 Jan 31 & Reticon & $2.5^{\prime \prime}$ & 124.5 & 0.151 & 0.484 \\
\hline NGC $5548 \ldots \ldots$. & 1984 Feb 4 & IDS & $7^{\prime \prime}$ & 125.0 & 0.134 & 0.446 \\
\hline NGC $5548 \ldots \ldots$. & 1984 Feb 6 & IDS & $7^{\prime \prime}$ & 120.6 & 0.161 & 0.464 \\
\hline
\end{tabular}

within the errors. There is thus no compelling reason to believe that any of the data are bad.

If indeed the observed difference between the Reticon and IDS scans is real, then continnum variability on the order of one day is implied and should be looked for in the future. It is at least somewhat reassuring that the $\mathrm{H} \beta$ flux did not show a similar change on the same time scale.

The closely spaced (i.e., $\Delta t \leq 30$ day) data from late 1983 and early 1984 clearly demonstrate that the broad $\mathrm{H} \beta$ flux responds to continuum changes on time scales shorter than one month.

In Figure 3, the $V$ magnitudes listed in Table 2 are shown as a function of Julian Date on the same scale as Figure 2. The simultaneous IDS and $U B V$ data points on 1984 March 3 (obtained at the same time with telescopes separated by less than $100 \mathrm{~m}$ ) can be used to set the zero point of Figure 2 at $14.61 \mathrm{mag}$ in $V$. If this is done, the 1983 January photometry agrees well with the IDS data. Furthermore, the 1984 April photometry is in fairly good agreement with the continuum decrease determined from the Reticon spectrum.

The only surprise from the photometry is the decrease in the continuum in mid-January 1984 and, based on the spectroscopy, its sudden recovery by the end of the month. This may explain why the $\mathrm{H} \beta$ flux in late January and early February is rather lower than expected from the optical continuum measurements made at the time-if the light-travel time for the BLR is of order 2 weeks, portions of the BLR had not yet responded to the continuum change by the time the spectroscopic measurements were obtained. Detailed analysis of the line profile is currently under way to determine if this might be the case.
Continuum and $\mathrm{H} \beta$ magnitudes obtained from spectroscopic measurements since late 1980 are shown in Figure 4. Adjacent points are connected with a straight line only to guide the eye from point to point, and, because the time scale for variability is apparently smaller than the typical temporal spacing of observations, the line provides only the crudest approximation to a light curve. It is nevertheless very clear from Figure 4 that the changes in the continuum flux have been accompanied by changes in the $\mathrm{H} \beta$ flux for at least $4 \mathrm{yr}$.

\section{DISCUSSION}

From the rapid response of $\mathrm{H} \beta$ to changes in the optical continuum flux, it can be inferred that the size of the BLR is quite small (of order $30 \mathrm{lt}$-day in radius). This is not the first evidence for a small BLR-Cherepashchuk and Lyutyi (1973) found that $\mathrm{H} \beta$ in NGC 4151, NGC 3516, and NGC 1068 followed changes in the optical continuum by less than one month. The short time-scale response of the BLR in NGC 4151 has been underscored by the observations of Antonucci and Cohen (1983) and of Bochkarev (1984).

The rapid response of the permitted lines to changes in the optical continuum leads to a number of problems in understanding the nature of the BLR. One is forced to conclude that the size of the BLR is small unless a contrived geometry is adopted, such as one in which essentially all the BLR gas lies along our line of sight to the continuum source. Unblended broad-line profiles are generally logarithmic in form, and the authors are unaware of any reasonable model for producing logarithmic profiles without requiring spherical or cylindrical symmetry (Capriotti, Foltz, and Byard 1980; Hubbard and Puetter 1983).

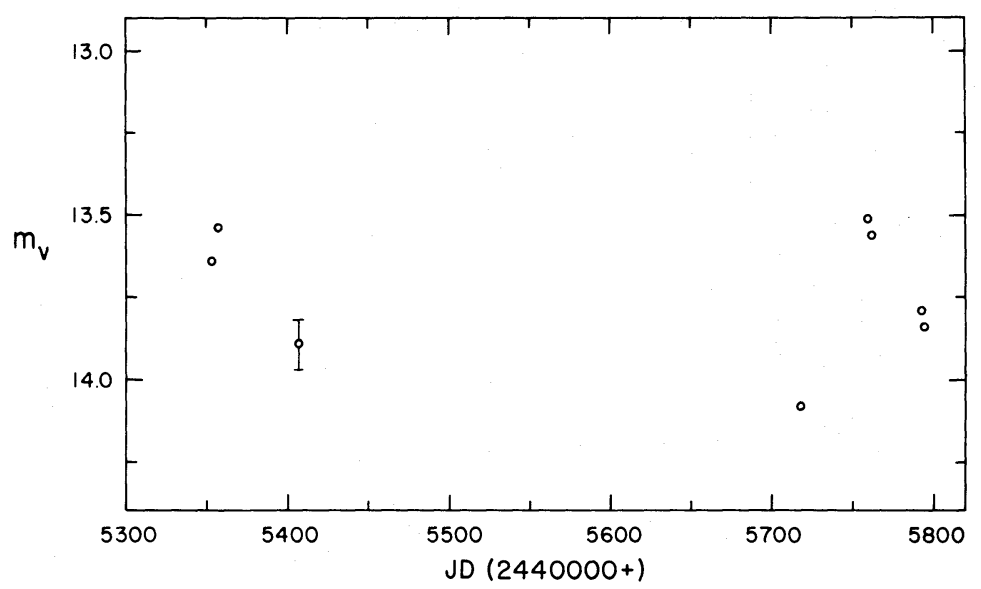

FIG. 3.-The apparent $V$ magnitudes listed in Table 2 are shown as a function of Julian Date 


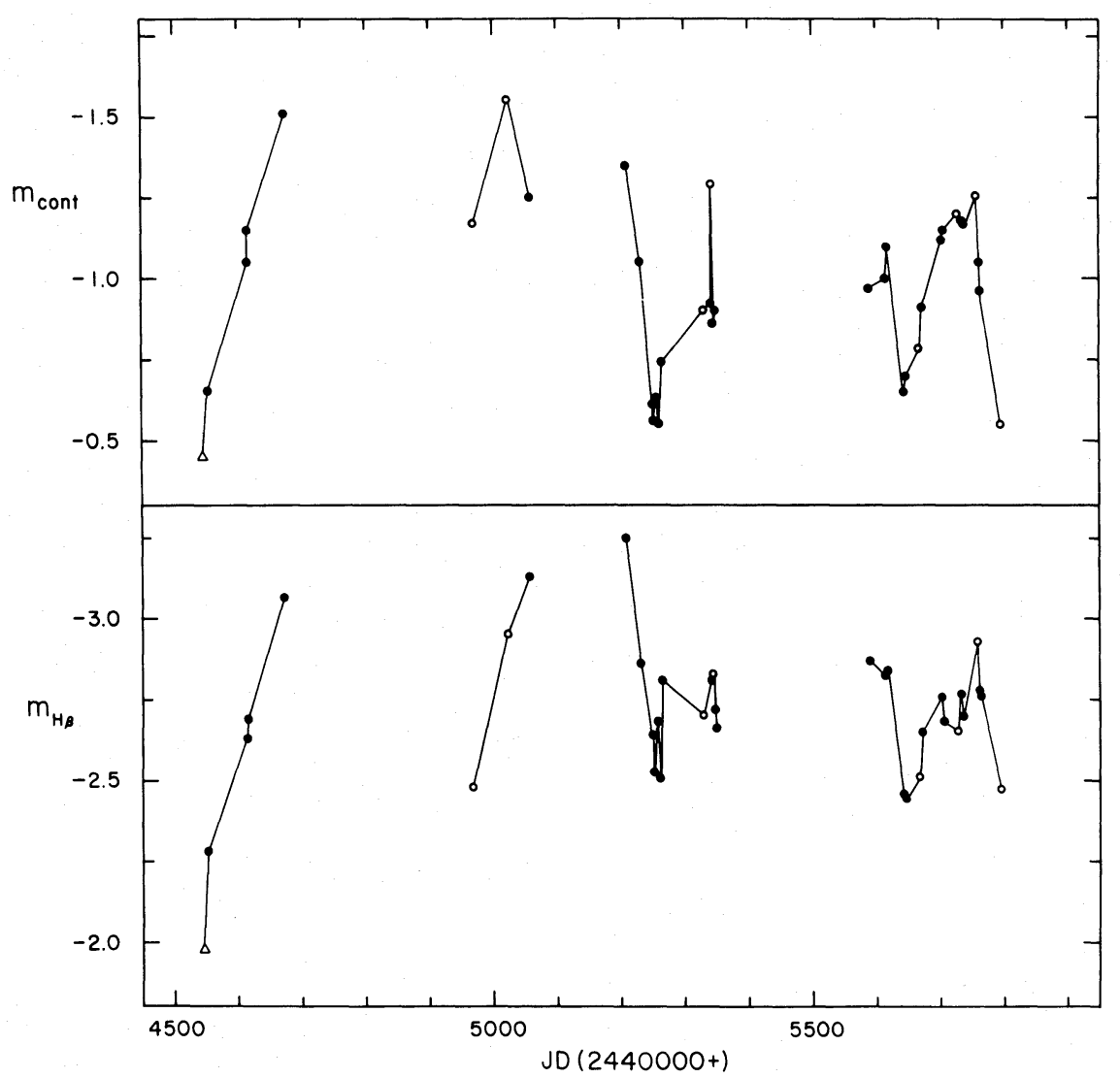

Fig. 4.-Optical continuum and $\mathrm{H} \beta$ magnitudes for all spectroscopic measurements since 1980, as a function of Julian Date. As in Fig. 1, the vertical scales are arbitrary, the closed circles represent IDS data, and the open circles represent Reticon data. The triangle is an estimate based on data from Kollatschny et al. (1981a). Adjacent points are connected to guide the eye.

The $\mathrm{H} \beta$ luminosity and electron density of the BLR set a limit on the minimum size of the BLR. For a case B recombination spectrum,

$$
r=0.0041\left(\frac{L_{42}}{N_{9}{ }^{2} \epsilon_{B}}\right)^{1 / 3} \mathrm{pc},
$$

where $L_{42}$ is the $\mathrm{H} \beta$ luminosity in units of $10^{42} \mathrm{erg} \mathrm{s}^{-1}, N_{9}$ is the electron density in units of $10^{9} \mathrm{~cm}^{-3}$, and $\epsilon_{B}$ is the filling factor for the BLR. For Akn $120, L_{42}$ and $N_{9}$ are both of order unity, so a very small BLR $(r \approx$ light-days) is possible from this standpoint.

The major difficulty with a small BLR surrounding the continuum source is that the radiation density in the BLR must be very high. For photoionization by a power-law continuum, the emission-line region can be characterized by the radiation parameter

$$
\Gamma=\int_{v_{1}}^{\infty} \frac{L_{v} d v}{4 \pi r^{2} c h v N_{e}}
$$

(McKee and Tarter 1975), where $L_{v}$ is the specific luminosity of the continuum source, $N_{e}$ is the electron density, and $v_{1}$ is the frequency at 1 ryd. Thus $\Gamma$ is the ratio of the ionizing photon density to the electron density in the BLR. Photoionization models that produce Seyfert 1 or QSO spectra generally have $\Gamma<1$; the most successful model at this time (Kwan and Krolik 1981) uses $\Gamma \approx 0.03$. For Akn 120 , the flux at 1 ryd can be estimated as $\sim 1.5 \times 10^{-26}$ ergs s${ }^{-1} \mathrm{~cm}^{-2} \mathrm{~Hz}^{-1}$ from the composite UV-X-ray spectrum of Kollatschny et al. (1981b) based on their IUE spectra and the X-ray data of Tananbaum et al. (1978) and Mushotzky and Marshall (1980). For $H_{0}=75$ $\mathrm{km} \mathrm{s}^{-1} \mathrm{Mpc}^{-1}$, it is found that

$$
\Gamma \approx \frac{1900}{N_{9} r_{d}^{2}},
$$

where $r_{d}$ is the size of the BLR in light-days. For $r_{d} \leq 30$, $\Gamma \geq 2 N_{9}^{-1}$, which implies that the electron density must be rather larger than currently supposed if $\Gamma$ is to be kept small enough to ensure a Seyfert 1 spectrum (if $\Gamma$ is increased to order unity, very high ionization lines which are not observed in the $I U E$ spectra ought to become prominent). The presence of C III] $\lambda 1909$ in the Akn 120 spectrum (Kollatschny et al. $1981 b$ ) argues that the electron density must be less than $\sim 10^{9.6} \mathrm{~cm}^{-3}$, and if indeed the shelf redward of $\mathrm{H} \beta$ is attributable at least in part to [O III] $\lambda \lambda 4959,5007$ from the BLR, an electron density perhaps an order of magnitude smaller than this is appropriate (Meyers and Peterson 1985).

There are at least three possible ways out of this dilemma. First, perhaps the flux at $h v>1$ ryd is lower than expected from an extrapolation of the far UV continuum. Suppression of this continuum does not seem consistent with observations of high redshift QSOs, however. In those QSOs in which the Lyman continuum is weak, it is probably because our line of sight passes through an optically thick broad-line emitting cloud or a galactic disk.

Second, it can be supposed that the electron density is large in the part of the BLR that produces the $\mathrm{H} \beta$ emission, and that 
the C III] $\lambda 1909$ (and also the [O III] $\lambda \lambda 4959,5007$ ) emission arises in a distinct region of lower electron density which is either farther away from the continuum source or optically thin. One then must explain why the C III $\lambda 1909$ profile has approximately the same width as the Balmer lines. On the other hand, Ulrich et al. (1984) find that in NGC 4151, C III] $\lambda 1909$ remains constant while $\mathrm{Mg}$ II $\lambda 2798$ and $\mathrm{C}$ IV $\lambda 1550$ respond rapidly to continuum changes, which implies that $\mathrm{C}$ III] $\lambda 1909$ arises in a region farther away from the continuum source. It seems fairly reasonable to assume that this may also be the case in Akn 120, and that the electron density in the Balmer line emitting region is greater than $\sim 5 \times 10^{10} \mathrm{~cm}^{-3}$.

An alternative explanation is given by Hubbard and Puetter (1983), who argue for high electron densities in the BLR despite the presence of strong $\mathrm{C} \mathrm{III}] \lambda 1909$. These authors note that if the BLR clouds are optically thick in C III] $\lambda 1909$ and the transition is dominated by collisions, the $\mathrm{C} \mathrm{III]/Ly} \alpha$ ratio is close to the observed value independent of density. In this case, there is no problem with requiring a higher electron density in the BLR.

Third, it might be supposed that the changes in the optical continuum lag behind changes in the ionizing continuum. Either this could be a property of the continuum source, or the optical continuum might originate in a region physically distinct from the ionizing continuum, but whose emission characteristics are sensitive to the ionizing continuum. This is a suggestion that might be worth following up on the observational and theoretical fronts. On the other hand, it is clear that if spherical symmetry of the BLR is to be preserved, the lighttravel time across the BLR must still be rather small, since short-term continuum variations will tend to average out in an extended BLR.

Another problem in the case of Akn 120 is that the Balmerline emission is slightly redshifted relative to the systemic velocity. It is possible to define a reflection point (redshift) about which each of the Balmer lines is fairly symmetric, except near line center and in the far wings of $\mathrm{H} \beta$. This reflection point is displaced redward of the systemic redshift (as determined from the $[\mathrm{O}$ III $] \lambda \lambda 4959,5007$ narrow lines and the weak $\mathrm{Mg}$ I b and $\mathrm{Na}$ I $\mathrm{D}$ absorption lines that presumably arise in the underlying galaxy) by $c \Delta z \approx 400 \mathrm{~km} \mathrm{~s}^{-1}$ (Foltz, Wilkes, and Peterson 1983; Meyers and Peterson 1985). Such redward displacements of the Balmer lines are common in active galaxies. There are several mechanisms that can easily lead to small displacements of the line peak or centroid or both, such as attenuation within the BLR (Ferland, Netzer, and Shields 1979; Capriotti, Foltz, and Byard 1979) or light-travel time effects (Capriotti, Foltz, and Peterson 1982). In the case of Akn 120 , the small size of the BLR affords an interesting explanation: if indeed the BLR surrounds a massive central object, the broad-line emission can be gravitationally redshifted. The required mass to produce the observed redshift would be

$$
M \approx \frac{c^{2} \Delta z r}{G} \approx 2.3 \times 10^{7} r_{d} M_{\odot},
$$

where again $r_{d}$ is measured in light-days. This is at least an order of magnitude greater than the minimum mass required by the Eddington limit,

$$
M_{\mathrm{E}} \approx 10^{6} L_{44} M_{\odot}
$$

where $L_{44}$ is the luminosity of Akn 120 in units of $10^{44}$ ergs $\mathrm{s}^{-1}$. Yee (1980) finds that the nonthermal luminosity of Seyferts and QSOs is typically $\sim 80 L_{\mathrm{H} \beta}$, which means $L_{44}$ is of order unity.

If the BLR is small and surrounds a collapsed massive object, then large emission-line widths are expected if equipartition applies. If the BLR clouds are in random motion, then $v^{2} \approx G M / r$, and from equation (6),

$$
v \approx c(\Delta z)^{1 / 2} \text {. }
$$

For $c \Delta z=400 \mathrm{~km} \mathrm{~s}^{-1}, v \approx 10^{4} \mathrm{~km} \mathrm{~s}^{-1}$, which compares favorably with $v_{\text {FWHM }}=6200 \mathrm{~km} \mathrm{~s}^{-1}$ for Akn 120 .

A gravitational redshift is an attractive way of explaining the slight redward displacement of the Balmer lines which is often observed in active-galaxy spectra. Clearly a measureable gravitational redshift must occur if active nuclei contain massive objects (eq. [7]) and the BLR is as small as it appears to be in Akn 120. Furthermore, if the virial theorem applies, a relationship between line width and redward displacement (eq. [8]) is predicted. As noted above, however, attenuation within the BLR or light-travel time effects can cause a displacement of the line peak and centroid, so a gravitational redshift is not necessarily required. An additional complication is that in higher redshift QSOs, Ly $\alpha$ and higher ionization lines like C IV $\lambda 1550$ are apparently blueshifted relative to the low ionization lines (Gaskell 1982; Wilkes 1984). However, C IV $\lambda 1550$ and C III] $\lambda 1909$ appear to arise in the same region, based on the similarity of their profiles in QSOs (Baldwin and Netzer 1978; Richstone, Ratnatunga, and Schaeffer 1980), and one might then conclude that C IV $\lambda 1550$ and C III] $\lambda 1909$ arise from farther out than do the Balmer lines and thus are closer to systemic velocity (i.e., less gravitationally redshifted). There are clear indications that such a model is naive, since the $\mathrm{C}$ III] profiles are sometimes clearly different from the $\mathrm{C}$ IV profiles (Wilkes 1984), although some of the differences may be attributable to contamination of the $\mathrm{C}$ III] profile by other lines, and in NGC 4151, the C IV line responds more rapidly than the $\mathrm{Mg}$ II line to changes in the continuum flux, which implies that the $\mathrm{C}$ IV emitting region is closer to the continuum source than the Mg II (and Balmer-line) emitting region (Ulrich et al. 1984). Unfortunately, existing UV spectra are not adequate for determination of the $C_{\text {IV }}$ and $C$ III] redshifts in Akn 120.

It should also be noted that the small size of the BLR places severe lifetime restrictions on certain kinematic models of the BLR. For example, models incorporating radial motion are now faced with a major difficulty. For the highest radialvelocity matter in Akn 120 (at $\sim 5000 \mathrm{~km} \mathrm{~s}^{-1}$ ), the crossing time for the BLR is

$$
\tau_{\mathrm{cr}}=\frac{R}{v} \approx 0.16 r_{d} \mathrm{yr}
$$

which is less than $5 \mathrm{yr}$ even if $r_{d}$ is as large as 30 lt-day. Akn 120 has been known to be variable for at least $55 \mathrm{yr}$, and over at least the last 4 yr the $\mathrm{H} \beta$ flux has rapidly responded to continuum changes. For a steady-state model with radial motion to remain viable, one must require that the characteristics of emitting clouds change rather abruptly at some small distance from the continuum source--within this distance, broad Balmer-line emission is observed, but beyond this distance it is absent or inconsequentially weak.

A restriction on virialized models, which was brought to our attention by Dr. W. G. Mathews, is that the lifetime of BLR clouds is limited by the mean time between cloud collisions

$$
\tau_{\text {coll }} \approx \frac{0.05 r_{d}}{f} \mathrm{yr}
$$


where $f$ is the covering factor for the BLR (i.e., the fraction of the sky covered by BLR clouds as seen from the center of the system). For $r_{d}<30, \tau_{\text {coll }}<1.5 f^{-1} \mathrm{yr}$, which is quite short unless $f$ is very small.

\section{SUMMARY}

On the basis of $4 \mathrm{yr}$ of spectroscopic monitoring of the Seyfert 1 galaxy Akn 120, several specific conclusions can be reached:

1. The BLR clouds are optically thick in the Lyman continuum, since the Balmer line emission varies with the continuum.

2. There is no perceptible time delay between continuum variations and $\mathrm{H} \beta$ variations, with temporal resolution sometimes better than one month. An upper limit to the radius of the BLR of 30 lt-day $\left(7.7 \times 10^{16} \mathrm{~cm}\right)$ is conservatively adopted.

3. Reasonable estimates of the ionizing flux of Akn 120 lead to a BLR radiation parameter which is too large by $\sim 2$ orders of magnitude if the conventional value for the electron density $\left(N_{e}=10^{9} \mathrm{~cm}^{-3}\right)$ is used. It seems likely that the electron density is higher than this value and $(a)$ the $C$ III] $\lambda 1909$ arises in a distinct region or $(b) \mathrm{C} \mathrm{III]} \lambda 1909$ is thermalized.

4. The broad Balmer lines are displaced redward from the systemic velocity by $c \Delta z \approx 400 \mathrm{~km} \mathrm{~s}^{-1}$. It is suggested that this may be a gravitational redshift, which would imply a mass of less than $\sim 7 \times 10^{8} M_{\odot}$ for the central object.
5. The emission line width are consistent with equipartition in the BLR, but intercloud collisions provide a strong constraint on the lifetime of BLR clouds.

6. The BLR crossing time is less than $\sim 4 \mathrm{yr}$, which seriously restricts models involving radial motion.

B. M. P. and K. A. M. are grateful to the National Science Foundation for support under grants AST 81-17095 and AST 83-17460 to Ohio State University. C. B. F. acknowledges the support of the Research Board of the University of Illinois. B. J. W. wishes to acknowledge the UK Science and Engineering Research Council for support on a NATO research fellowship. H. R. M. was supported in part by a grant from the Vice President's Research Fund at Georgia State University. Special thanks are due to Dr. Paul L. Byard and Mr. Craig Gullixson for their efforts in maintaining the high level of performance of the IDS. Drs. R. J. Weymann and M. Malkan are thanked for obtaining the 1984 February 29 spectrum at the MMT, and Mr. Howard A. Bushouse is thanked for obtaining the 1984 April 6 Reticon spectrum. Enlightening conversations with several participants of the Seventh Santa Cruz Workshop on Astronomy and Astrophysics, particularly W. G. Mathews and R. C. Puetter, are gratefully acknowledged.
Antonucci, R. R. J., and Cohen, R. D. 1983, Ap. J., 271, 564.

Baldwin, J. A., and Netzer, H. 1978, Ap. J., 226, 1.

Blandford, R. D., and McKee, C. F. 1982, Ap. J., 255, 419.

Bochkarev, N. 1984, preprint.

Byard, P. L., Foltz, C. B., Jenkner, H., and Peterson, B. M. 1981, Pub. A.S.P 93, 147 .

Capriotti, E., Foltz, C., and Byard, P. 1979, Ap. J., 230, 681

. 1980, Ap. J., 241, 903.

Capriotti, E. R., Foltz, C. B and Peterson, B. M. 1982, Ap. J., 261, 35.

Cherepashchuk, A. M., and Lyutyi, V. M. 1973, Ap. Letters, 13, 165.

Ferland, G. J., Netzer, H., and Shields, G. A. 1979, Ap. J., 229, 274.

Foltz, C. B., Peterson, B. M., Capriotti, E. R., Byard, P. L., Bertram, R., and

Lawrie, D. G. 1981, Ap. J., 250, 508.

Foltz, C. B., Wilkes, B. J., and Peterson, B. M. 1983, A.J., 88, 1702.

Gaskell, C. M. 1982, Ap. J., 263, 79.

Hubbard, E. N., and Puetter, R. C. 1983, Ap. J., 265, 35.

Kollatschny, W., Fricke, K. J., Schleicher, H., and Yorke, H. W. 1981a, Astr.

Ap., 102, L23. 1981b, Astr. Ap., 104, 198

Kwan, J., and Krolik, J.H. 1981, A.J., 250, 478.

McKee, C. F., and Tarter, C. B. 1975, Ap. J., 202, 306.

Meyers, K. A., and Peterson, B. M. 1985, $A p$. J., submitted.

Miller, H. R. 1979, Astr. Ap. Suppl., 35, 387.

\section{REFERENCES}

Miller, H. R. 1981, A.J., 86, 87.

Mushotzky, R. F., and Marshall, F. E. 1980, Ap. J. Letters, 239, L5.

Osterbrock, D. E., and Phillips, M. M. 1977, Publ. A.S.P., 89, 251.

Osterbrock, D. E., and Shuder, J. M. 1982, Ap. J. Suppl., 49, 149.

Peterson, B. M., and Collins, G. W., II. 1983, Ap. J., 270, 71.

Peterson, B. M., Foltz, C. B., Byard, P. L., and Wagner, R. M. 1982, Ap. J. Suppl., $49,469$.

Peterson, B. M., Foltz, C. B., Crenshaw, D. M., Meyers, K. A., and Byard, P. L. 1984, Ap. J., 279, 529.

Peterson, B. M., Foltz, C. B., Miller, H. R., Wagner, R. M., Crenshaw, D. M., Meyers, K. A., and Byard, P. L. 1983, A.J., 88, 926 (Paper I).

Phillips, M. M. 1978, Ap. J. Suppl., 38, 187 .

Phillips, M. M., Baldwin, J. A., Atwood, B., and Carswell, R. F. 1983, Ap. J., 274, 558.

Richstone, D. O., Ratnatunga, K., and Schaeffer, J. 1980, Ap. J., 240, 1.

Schulz, H., and Rafanelli, P. 1981, Astr. Ap., 103, 216.

Tananbaum, H., Peters, G., Forman, W., Giacconi, R., Jones, C., and Avni, Y. 1978, Ap. J., 223, 74

Ulrich, M. H., et al. 1984, M.N.R.A.S., 206, 221.

Walker, M. F. 1968, Ap. J., 151, 71.

Whittle, D. M. 1981, Ph.D. thesis, Cambridge University

Wilkes, B. J. 1984, M.N.R.A.S., 207, 73 .

Yee, H. K. C. 1980, Ap. J., 241, 894.

E. R. Capriotti, K. A. Meyers, and B. M. Peterson: Department of Astronomy, The Ohio State University, 174 W. 18 th Ave., Columbus, $\mathrm{OH} \quad 43210$

C. B. Foltz: Multiple Mirror Telescope Observatory, University of Arizona, Tucson, AZ 85721

H. R. MiLler: Department of Physics and Astronomy, Georgia State University, Atlanta, GA 30303

B. J. Wilkes: Center for Astrophysics, Smithsonian Astrophysical Observatory, 60 Garden Street, Cambridge, MA 02138 